\title{
Maximizing Output Power of a CFPG Micro Energy- Harvester for Wearable Medical Sensors
}

\author{
Mehdi Dadfarnia ${ }^{1}$, Kamran Sayrafian ${ }^{2}$, Paul Mitcheson ${ }^{3}$, John S. Baras ${ }^{1}$ \\ ${ }^{1}$ Institute for Systems Research and Electrical \& Computer Engin. Dept. \\ University of Maryland, College Park, USA \\ ${ }^{2}$ Information Technology Laboratory \\ National Institute of Standards \& Technology, Gaithersburg, USA \\ ${ }^{3}$ Department of Electrical \& Electronic Engineering \\ Imperial College London, London, UK
}

\begin{abstract}
-Abstract-Energy Harvesting refers to the process of capturing and storing energy from the ambient environment. Kinetic energy harvested from the human body motion seems to be one of the most convenient and attractive solutions for wearable wireless sensors in healthcare applications. Due to their small size, such sensors are often powered by small batteries which might necessitate frequent recharge or even sensor replacement. Energy harvesting can prolong the battery lifetime of these sensors. This could directly impact their everyday use and significantly help their commercial applications such as remote monitoring. In this paper, our aim is to develop a Simulink model of the CFPG device that can be used to study temporal behavior of the generated power. Having such a dynamic model, not only helps to have a more accurate estimation of the amount of power generated from various human movements, but also allows us to further optimize the design parameters of the micro-harvester (e.g. size/dimension, electrostatic holding force, etc.) with the characteristics of the input acceleration (i.e. human activity).
\end{abstract}

Index Terms-Micro energy-harvester, body sensors, mathematical modelling

\section{INTRODUCTION}

Wearable medical sensors have become a promising interdisciplinary research area in pervasive health information technology. However, various challenging issues including miniaturized sensing/actuator technology, security, reliability and power efficiency still remain. Wireless wearable sensors offer an attractive set of e-health applications among which we can point to various medical \& physiological monitoring such as temperature, respiration, heart rate, and blood pressure [2]. As these sensors mainly rely on very small batteries to carry their functions, prolonging their operational lifetime could significantly help their successful commercial application.

Energy Harvesting (EH) refers to the process of capturing and storing energy from the ambient environment. There are

The work of M. Dadfarnia was supported by the National Institute of Standards and Technology (NIST) through grant 70NANB11H148 and through an internship. The work of J.S. Baras was partially supported by (NIST) grant 70NANB11H148 and by NSF grant CNS1035655.

${ }^{1}$ Simulink is a product of MathWorks, Inc. Simulink has been used in few sources from which we can harvest energy for wearable medical sensors; amongst them are light, body heat and typical movements of the human body. Kinetic energy harvested from human body motion seems to be one of the most convenient and attractive solutions for wearable wireless sensors in healthcare applications.

Miniaturized energy harvesting devices, also known as micro-generators, which harvest energy from kinetic motion consists of a mass-spring-damper (MSD), a transducer, and an interfacing power-processing circuit as depicted in Figure 1 [10].

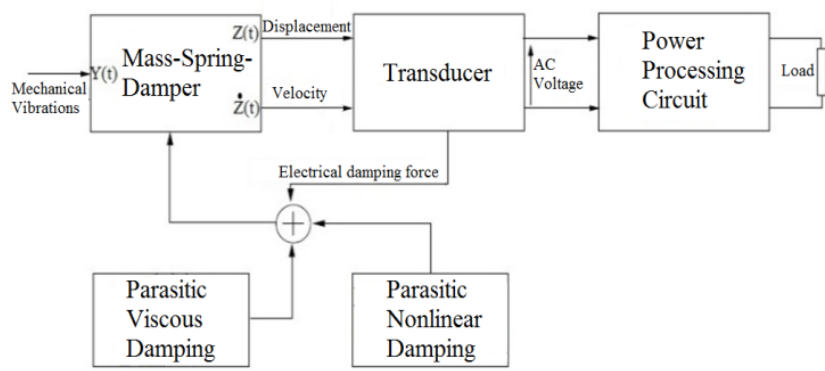

Fig. 1. Generic Electromechanical Block Diagram of an Inertial Microgenerator [10]

Kinetic microgenerators either utilize direct application of force on the device or they make use of inertial, ambient forces acting on a proof mass. These forces are captured with the MSD component. Unlike microgenerators that utilize a direct application of force, their inertial counterparts require only one point of attachment to the moving structure. This allows for greater mounting flexibility and also a greater degree of miniaturization that is ideal for wearable sensors. A generic model of such a MSD system is depicted in Figure 2 [1]. In this model, the displacement of the mass from its rest position relative to the frame is denoted by $z(t)$. The absolute motion of the frame is $y(t)$ and that of the proof mass is $x(t)=y(t)+$ $z(t)$. The proof mass is able to move between the upper and lower bounds i.e. $+/-Z_{l}$, and is attached to a spring-like structure with spring constant $k$. Energy is converted when 
work is done against the transducer's damping force $f\left(z^{\prime}\right)$, which opposes the motion based on direction of proof mass velocity, $z^{\prime}(t)[1,4]$. The micro-generator needs to be able to efficiently harvest energy from the human body motion. Most MSD designs for microgenerators in the literature use a spring or spring-like feature. This gives the device an intrinsic resonant frequency that is dependent on the spring constant, and makes the microgenerator most suitable for applications where the environment causes the MSD system to constantly vibrate [5]. However, human body motion is typically not a fixed frequency vibrating source motion. As a result, a microgenerator that can efficiently convert energy from human body motion into an electrical form should be designed to be non-resonating [8].

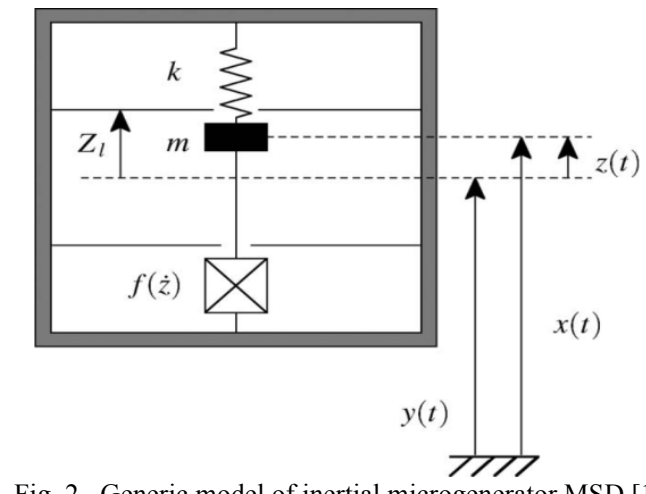

Fig. 2. Generic model of inertial microgenerator MSD [1]

One such non-resonating micro-generator architecture is the Coulomb-Force Parametric Generator (CFPG) [7]. The MSD system of this design is non-linear in nature. The proof mass doesn't vibrate up and down as if anchored on a springlike structure. Instead the transducer's damping force, a constant Coulomb electrostatic holding force, keeps the proof mass to an end-stop limit of $+/-Z_{l}$. The proof mass is held against one end-stop until the external acceleration exceeds the holding force threshold, thus ensuring that the distance between the end-stop limits is traveled by the proof mass under the highest possible force [8]. No power is generated while the proof mass is stuck on either side. Power is generated when the proof mass makes a full flight from one end-stop to the other.

The authors in [3] provided a methodology to estimate the amount of the average generated mechanical power from typical human motion using a mathematical model of the CFPG architecture. One of our contributions in this paper is an enhanced Simulink ${ }^{1}$ model of the CFPG device that can be used to study temporal behavior of the generated power. Having such a dynamic model, not only helps to have a more accurate estimation of the amount of power generated from

${ }^{1}$ Simulink is a product of MathWorks, Inc. Simulink has been used in this research to foster research and understanding. Such identification does not imply recommendation or endorsement by the National Institute of Standards and Technology, nor does it imply that this product is necessarily the best available for the purpose. various human movements, but also allows us to further optimize the design or operational parameters of the microharvester (e.g. size/dimension, electrostatic holding force, etc.) with the characteristics of the input acceleration.

The remainder of this paper is organized as follows. Section II discusses the mathematical modeling and Simulink implementation of the MSD model with sample preliminary results of our developed model for sample human arm motion, In section III, we describe an optimization problem that relates the input acceleration, the electrostatic holding force, and the output generated power. Finally, conclusions and future work are briefly discussed in Section IV.

\section{Mathematical Modeling \& SimUlinK IMPLEMENTATION}

The following non-linear differential equation has been specified as a model to capture the dynamics of the MSD module in a CFPG micro energy harvester [7].

$$
m y^{\prime \prime}(t)=-m z^{\prime \prime}(t)-F * \operatorname{sign}\left(z^{\prime}(t)\right) .
$$

In the above equation, $m$ represents the proof mass, $y(t)$ is the motion of the generator frame with respect to the inertial frame $\left(y^{\prime \prime}(t)\right.$ is the second derivative of $y(t)$ and indicates the input acceleration), $z^{\prime \prime}(t)$ is the proof mass acceleration, $F$ represents the Coulomb force (also referred to as electrostatic holding force or more generally the MSD's damping force), and $\operatorname{sign}\left(z^{\prime}(t)\right)$ represents the sign (or equivalently direction) of the proof mass velocity (i.e. $z^{\prime}(t)$. The generated mechanical power by the MSD component can be computed by Eq. (2) as follows:

$$
P(t)=F * Z^{\prime}(t)
$$

where $F$ is the holding force and $z^{\prime}(t)$ represents the velocity of the proof mass.

Although Eq. (1) mathematically captures the simple conceptual function of the MSD component, there are several constraints that make the direct implementation of this equation more complicated. These constraints represent the physical laws that determine how and when energy is generated by the proof mass movements. For example, energy is only generated when the proof mass makes a complete flight between the end-stops of the MSD frame (i.e. $\left.+/-Z_{l}\right)[7$, 8]. This implies that the output of the sign function in Eq. (1) should only change when this occurs. However, this fact is not evident by the current form of this mathematical equation.

As stated previously, our first objective is to have an accurate model that can simulate the instantaneous power generated by the CFPG device. To achieve this, we used Simulink to create an implementation of the MSD model and made sure that mathematically it satisfies all physical constraints that impact the generated power. In doing so, we realized that the direct implementation of Eq. (1), (with the $\operatorname{sign}($.$) function) does not result in a model that satisfies the$ 
physical criteria for generating energy. Therefore, we have modified the mathematical equation into the following form:

$$
m y^{\prime \prime}(t)=-m z^{\prime \prime}(t)-F * \operatorname{relay}(z(t))
$$

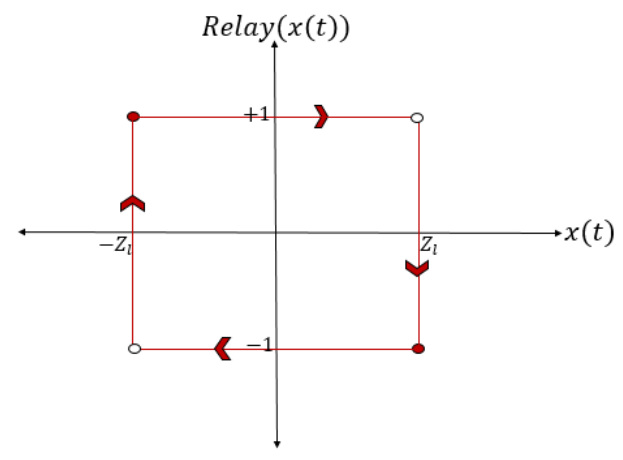

Fig. 3. Relay Function

The relay(.) in the above equation (3) models a hysteresis function and switches between $+/-1$ values. When the relay is ON (i.e. -1 ), it remains $\mathrm{ON}$ until the input (i.e. $z(t)$ ) hits the lower frame boundary i.e. $-Z_{l . .}$ At that point, it switches to OFF (i.e. +1$)$ and remains off until the $z(t)$ hits the upper boundary $\left(+Z_{l}\right)$. This function is graphically shown in Fig. 3. Note that, unlike the sign function in Eq. (1), the output of the relay function depends on the position of the proof mass rather than its velocity. However, the output reflects the changes in the direction of the proof mass velocity only when it reaches the end-stops of the frame. This will result in the correct mechanism for generating power from the MSD component. The Simulink implementation of this model is depicted in Figure 4. To verify the modified differential equation and our Simulink model, several test scenarios were generated. For brevity, we are omitting the description of those scenarios.

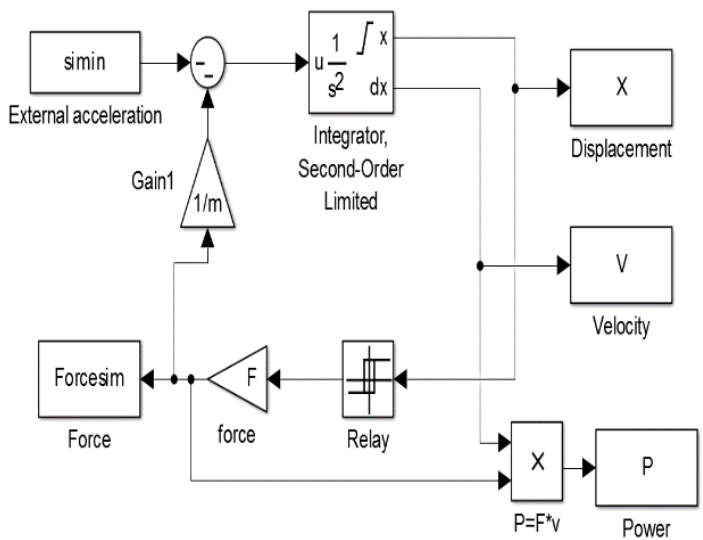

Fig. 4. Simulink model of CFPG MSD system

Using this Simulink model, the temporal behavior of the generated power can be studied for various input acceleration waveforms. For example, Fig. 5 shows a 60 seconds sample acceleration of human arm motion as obtained in [3]. By applying this acceleration to the Simulink model, we can observe the instantaneous power that is generated by the
CFPG device, see Figure 6. Note that if the proof mass does not make a complete flight to the opposite end stop, the energy that was put into the electric field is removed and hence the instantaneous power would be negative. The average harvested power for this sample acceleration is $15.944 \mu \mathrm{W}$. For this simulation, we have assumed a MSD with the following specifications: proof mass is $0.965 \mathrm{mg}$, and the distance between the two end-stops is $5 \mathrm{~mm}$.

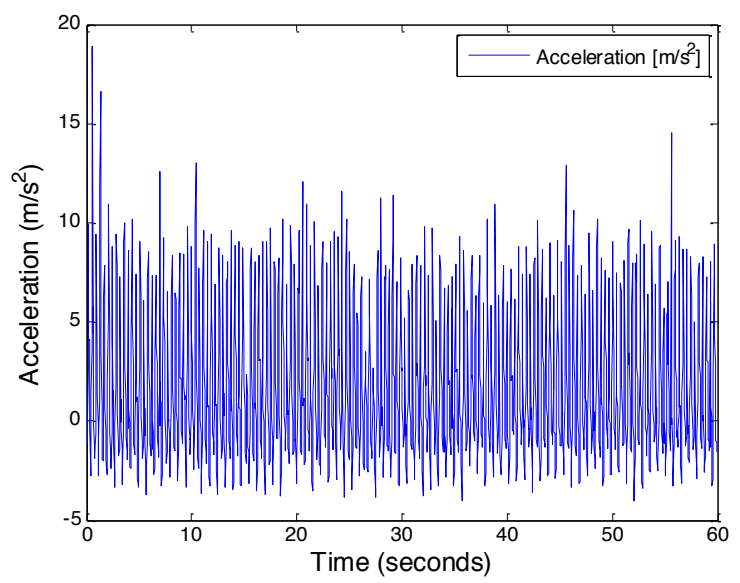

Fig. 5. 60-second acceleration sample of human arm motion

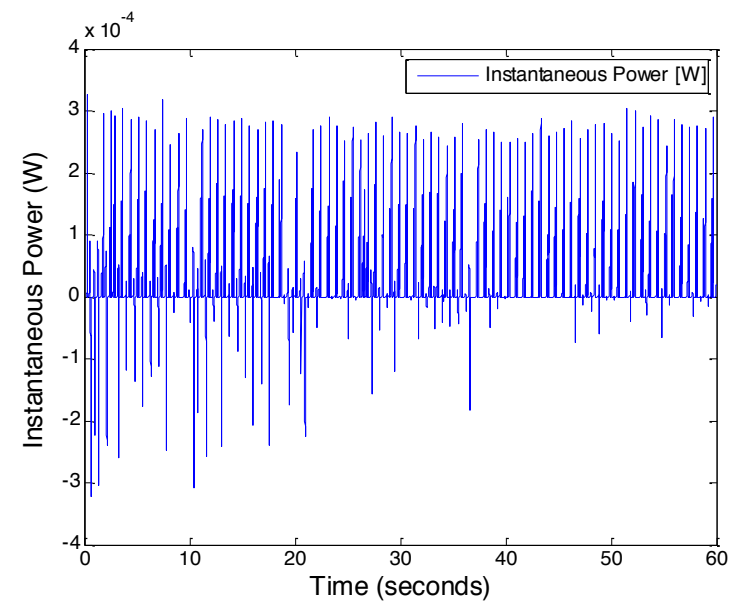

Fig. 6. Generated instantaneous power for human arm motion acceleration sample (see Fig. 5.) and a constant electrostatic holding force $\mathrm{F}=1.25 \mathrm{mN}$

\section{OPTIMIZATION OF THE HARVESTED POWER}

An important objective in our study is to characterize the impact of the electrostatic force ' $F$ ' on the amount of generated power. The results in Fig. 6 are obtained by using a constant $F$ (i.e. $1.25 \mathrm{mN}$ ) regardless of the input acceleration. Here, it is shown that by judiciously choosing the value of $F$, one can maximize the average generated power. For this purpose, the following optimization problem has been formulated:

$$
\operatorname{Argmax}_{F_{i}}\left[\left(\frac{1}{\Delta}\right) \times \sum_{t=t_{i}}^{\Delta+t_{i}} P(t)\right]
$$

such that 
and

$$
P(t)=F_{i} * z^{\prime}(t)
$$

$$
m y^{\prime \prime}(t)=-m z^{\prime \prime}(t)-F_{i} * \operatorname{relay}(z(t))
$$

This aims to maximize the average harvested power during the time interval $\Delta$ by choosing the optimal value of the electrostatic force $F$. The choice of $\Delta$ translates to how quickly the value of the electrostatic force should be adapted to the input in order to maximize the output power. For our preliminary studies in this paper, we have considered this length as another parameter and provided the results for various interval sizes i.e. $10,4,2,1,0.5,0.2$, and .125 seconds. To the best of our knowledge, this is the first time that such adaptive optimizations are being applied to such micro-harvesters.

Using the Pattern Search optimization algorithm [11], we have solved the above problem for the sample data shown in Fig. 5. Table 1 summarizes the results of this optimization. These results point to the potential impact of using the optimal value of the electrostatic force $F$ on the harvested power. As observed, a significant gain (almost 5 times higher) is achieved for the sample data compared to the non-optimized scenario. We also tested other sample acceleration data (representing natural human arm motion) as measured in [3]. Although the amount of the optimal average power could be different for different input acceleration waveforms, still noticeable gains in average harvested power are observed. For brevity, we are omitting those results.

Table 1. Optimal average power versus for sample data in Fig. 5

\begin{tabular}{|c|c|c|}
\hline $\begin{array}{c}\text { Optimization } \\
\text { Interval } \Delta \\
(\text { Sec })\end{array}$ & $\begin{array}{c}\text { Optimal Average } \\
\text { Harvested Power } \\
(\mu \mathrm{W})\end{array}$ & $\begin{array}{c}\text { Ratio of } \\
\text { Optimization/Non- } \\
\text { Optimization in Power } \\
\text { Gain }\end{array}$ \\
\hline $1 / 8$ & 78.3 & 4.19 \\
\hline $1 / 4$ & 45.9 & 2.88 \\
\hline $1 / 2$ & 33.9 & 2.13 \\
\hline 1 & 23.2 & 1.46 \\
\hline 2 & 20.5 & 1.29 \\
\hline 4 & 18.4 & 1.16 \\
\hline 10 & 18.2 & 1.15 \\
\hline
\end{tabular}

\section{CONCLUSIONS \& FUTURE WORK}

In this paper, we have presented a model of the MSD component based on a modified non-linear differential equation. This model can be used to measure the amount of mechanical power generated. The conversion to electrical power is done through the transducer module as reflected in Fig. 1. The authors plan to continue the development of a Simulink model for the transducer, and study the electrical power generated by various input excitations as well. Our ultimate goal in this research is to develop an accurate Simulink model of the CFPG micro energy-harvester device and optimize the amount of generated power for various human body motions.

Integration of micro energy harvesting technology with wearable sensors is a promising approach in prolonging the operational lifetime of wearable medical sensors. Optimizing the architectural and design parameters of the harvester device based on the characteristics of the input acceleration will increase the amount of the generated power. This is a prime example of a Cyber-Physical System (CPS) that highlights how joint design of the cyber and physical components can improve the system efficiency. By adaptively tuning the electrostatic force $F$ for various human body motions, one can expect an improved efficiency in harvesting kinetic energy for wearable sensors. Physically, this can be accomplished by tuning the electric field between the CFPG's capacitive electrodes [9]. Mathematical formulation of this problem involves a non-linear optimization problem that can be solved by adding appropriate computational algorithms in the microgenerator architecture. The authors plan to continue this research and study the impact of adaptive optimization on the harvestable power using a CFPG architecture.

\section{REFERENCES}

[1] Mitcheson, Paul D., Eric M. Yeatman, G. Kondala Rao, Andrew S. Holmes, and Tim C. Green. "Energy harvesting from human and machine motion for wireless electronic devices." Proceedings of the IEEE 96, no. 9 (2008): 1457-1486.

[2] Hao, Yang, and Robert Foster. "Wireless body sensor networks for health-monitoring application." Physiological measurement 29, no. 11 (2008): R27

[3] Yarkony, Nathalie, Kamran Sayrafian-Pour, and Antonio Possolo. "Statistical modeling of harvestable kinetic energy for wearable medical sensors." In World of Wireless Mobile and Multimedia Networks (WoWMoM), 2010 IEEE International Symposium on a, pp. 1-5. IEEE, 2010.

[4] Mitcheson, Paul D., Tim C. Green, Eric M. Yeatman, and Andrew S. Holmes. "Architectures for vibration-driven micropower generators."Microelectromechanical Systems, Journal of 13, no. 3 (2004): 429-440

[5] Kazmierski, Thomas J., ed. Energy Harvesting Systems: Principles Modeling and Applications. Springer, 2011

[6] Mitcheson, Paul D., Tom Sterken, Cairan He, M. Kiziroglou, E. M. Yeatman, and R. Puers. "Electrostatic microgenerators." Measurement and Control 41, no. 4 (2008): 114-119

[7] Mitcheson, Paul David. "Analysis and optimisation of energy-harvesting micro-generator systems." PhD diss., Imperial College, 2005

[8] Von Buren, Thomas, Paul D. Mitcheson, Tim C. Green, Eric M. Yeatman, Andrew S. Holmes, and Gerhard Troster. "Optimization of inertial micropower generators for human walking motion." Sensors Journal, IEEE 6, no. 1 (2006): 28-38.Matlab/Simulink 2013b.

[9] Mitcheson, Paul D., Tim C. Green, and Eric M. Yeatman. "Power processing circuits for electromagnetic, electrostatic and piezoelectric inertial energy scavengers." Microsystem Technologies 13, no. 11-12 (2007): 1629-1635.Miao mems inertial power generations

[10] Gandu, Kondalarao. "Power Processing for Electrostatic Microgenerators." PhD diss., Imperial College, 2011'

[11] Torczon, Virginia. "On the convergence of pattern search algorithms." SIAM Journal on optimization 7, no. 1 (1997): 1-25. 